\title{
Exploiting RET isoforms in managing medullary and papillary thyroid cancer
}

Eric Y Lian ${ }^{1}$, Serisha Moodley ${ }^{1}$ \& Lois M Mulligan*,1

'Division of Cancer Biology \& Genetics, Cancer Research Institute, \& Department of Pathology \& Molecular Medicine, Queen's University, Kingston, ON K7L 3N6, Canada

*Author for correspondence: Tel.: +1 613533 6000; Fax: +1 613533 6830; mulligal@queensu.ca

"The recent use of high throughput screening techniques has identified novel RET rearrangements in other tumors, suggesting roles for RET in the development and progression of multiple human cancers."

First draft submitted: 12 December 2017; Accepted for publication: 08 January 2018; Published online: 27 July 2018

Keywords: MTC • PTC • RET isoforms

The RET receptor mediates cell proliferation, survival and migration in embryogenesis, and is implicated in transformation and tumor progression in multiple cancers. RET is frequently mutated and constitutively activated in familial and sporadic thyroid carcinomas. RET is expressed as two isoforms, RET9 and RET51, that differ in C-terminal amino acids. These isoforms interact with distinct downstream signaling and regulatory complexes, but their functional differences are not well defined, and the effects of RET isoforms on disease prognosis and treatment remain poorly understood. In this editorial, we review the current understanding of differences between the RET isoforms and how they might affect disease course and clinical outcomes and serve as potential therapeutic targets in medullary or papillary thyroid carcinoma, and other RET-associated tumors.

Thyroid cancer affects over 3 million people worldwide. Papillary thyroid carcinoma (PTC) is the most commonly diagnosed thyroid malignancy, accounting for approximately $85 \%$ of all these cases, and is generally treatable, with a 5 -year survival rate of $98 \%$ [1]. Medullary thyroid carcinoma (MTC) is a less common subtype that makes up some $3-5 \%$ of all thyroid cancers, displays more aggressive phenotypes, and can be more difficult to manage [1]. Surgical removal of the thyroid, followed by postoperative thyroid hormone replacement therapy, contributes to successful disease management in the majority of both PTC and MTC cases [2]. However, a subset of patients experience recurrence and/or distant metastases [2,3]. To date, additional treatment strategies, such as external beam radiation or chemotherapy, have had limited efficacy and are associated with adverse effects $[2,3]$ posing an urgent need for predictive markers to distinguish treatment-resistant cases, as well as new treatment options for these persistent thyroid cancers. The advent of methodologies to target common oncogenic proteins or mutants that contribute to thyroid cancers is providing new therapeutic opportunities to address these challenging cases.

The rearranged during transfection (RET) receptor tyrosine kinase is a transmembrane tyrosine kinase expressed in many neuroendocrine tissues, nerves and the kidney [4]. RET is activated by binding of GDNF family ligands and GFR $\alpha$ family co-receptors (Figure 1) to mediate numerous cellular processes including cell survival, proliferation, migration, and differentiation [4]. However, RET is also an oncogene associated with growth and spread of cancer in multiple human tissues, including thyroid, where it promotes both MTC and PTC [5]. Activating point mutants of RET are associated with MEN2, a cancer syndrome characterized by early onset MTC, and similar mutations are also linked to poor clinical outcome in $40-65 \%$ of sporadic MTC [4]. These mutations result in ligand independent receptor dimerization or reduced autoinhibition and increased ATP-binding, leading to constitutive RET activity [4].

In PTC tumors, chromosomal rearrangement of the RET gene can generate RET chimeric proteins consisting of the RET kinase-domain fused to $\mathrm{N}$-terminal sequences of a different protein that contains homodimerization domains. These constitutively activated proteins are aberrantly localized to the cytosol [4,5]. The recent use of high throughput screening techniques has identified novel RET rearrangements in other tumors, most notably in lung
Future Medicine 
(A)

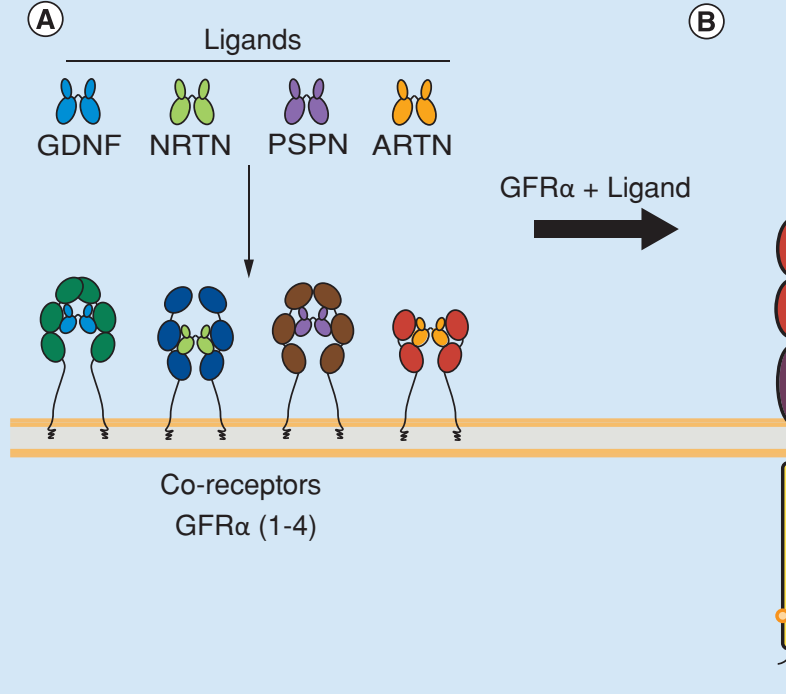

(B)

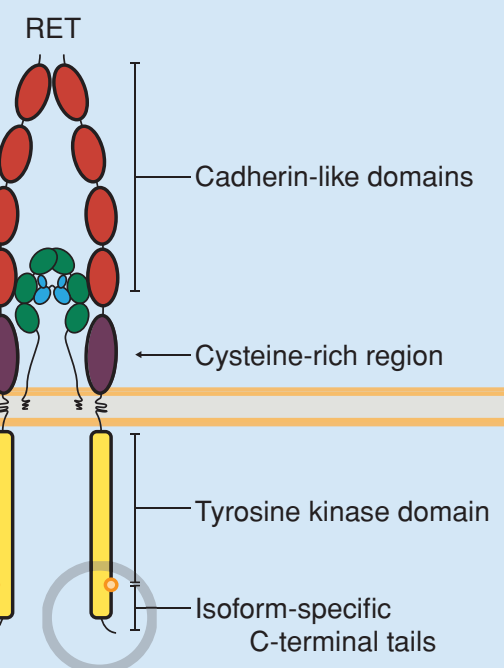

Alternative splicing

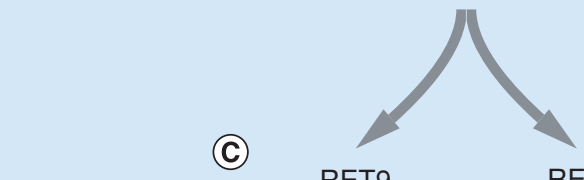

(C)

SH2-binding motif

PDZ-binding motif
RET51

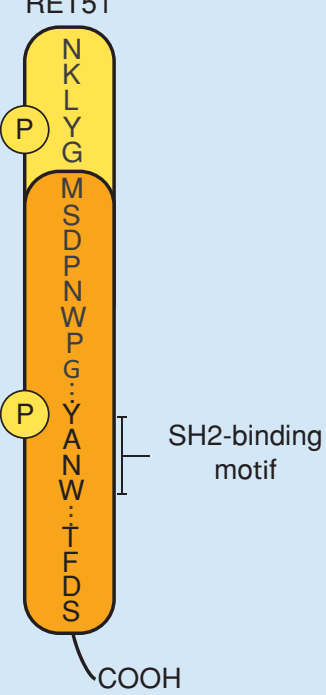

Figure 1. RET receptor tyrosine kinase structure and activation. (A) Activation of RET requires the formation of a multicomponent complex which involves a GDNF family ligand (GDNF, NRTN, ARTN or PSPN) and its respective co-receptor (GFR $\alpha$ 1-4). (B) RET is a transmembrane tyrosine kinase receptor with three main regions. The extracellular domain consists of four cadherin-like domains and a cysteine-rich region, linked to an intracellular tyrosine kinase domain and isoform-specific C-terminal tails. (C) RET has two main isoforms with unique nine or 51 amino acid (RET9 and RET51, respectively) C-terminal sequences. RET9 and RET51 possess different phospho-tyrosine and protein-binding motifs and display differential abilities to bind downstream adaptor and signaling molecules.

adenocarcinomas and chronic myelemonocytic leukemia, as well as in spitzoid tumors [4,6], suggesting roles for RET in the development and progression of multiple human cancers. 


\section{Differential functions of RET isoforms}

Our understanding of RET's roles in tumorigenesis, and its effects on disease progression, is complicated by the expression of two RET protein isoforms, generated by alternative splicing of $3^{\prime} R E T$ gene sequences. These proteins, termed RET9 and RET51, are identical, save for the unique nine or 51 amino acids in their respective C-terminal tails [7] (Figure 1). Despite the minor structural differences between these isoforms, a large body of evidence now suggests that RET9 and RET51 have very different contributions to RET-mediated cellular processes. Although RET9 and RET51 are coexpressed, the relative amounts of each isoform are temporally and spatially regulated, suggesting differences in their biological roles. The RET isoforms are known to mediate distinct processes during embryogenesis, with RET9, but not RET51, being critical for normal kidney morphogenesis and enteric nervous system development [8]. RET9 is highly expressed during early development, but RET51 expression remains low until later developmental stages, suggesting involvement in differentiation related events $[9,10]$. RET9 is also expressed ubiquitously throughout the olfactory nervous system, while RET51 expression is confined to regions innervated by sensory neurons [11], consistent with a more specialized role in directing cell migration, axon growth and guidance $[12,13]$.

More recently, we have begun to understand the molecular and biological mechanisms that contribute to these RET isoform-specific roles. RET isoforms recruit distinct signaling complexes, leading to different patterns of target gene expression [14,15]. Although both RET9 and RET51 promote downstream signaling through multiple pathways, including PI3K/Akt/mTOR, and ERK/MAPK, distinct protein interactions with RET isoform unique C-terminal sequences affect the timing, duration and subcellular localization of these events $[4,14,16,17]$. Most notably, RET51 interacts with adaptor proteins containing SH2 domains, such as GRB2, through a unique phosphorylated tyrosine residue (pY1096) in its C-terminal tail [16,18]. In contrast, RET9 has distinct binding capabilities at pY1062, and also contains a unique C-terminal binding motif $\left(\mathrm{FTRF}_{1072}\right)$, which allows it to interact with PDZ domain proteins such as SHANK family members [4,16] (Figure 1). RET9 and RET51 have been found to differ in their membrane localization, and in their internalization into the cell and intracellular trafficking upon activation $[16,17,19]$. RET51 matures more efficiently than RET9, and is present in relatively greater quantities at the cell membrane, while RET9 matures slowly and accumulates as immature protein [17,20]. Upon activation, RET51 is more rapidly internalized from the cell surface, but can be recycled from endosomal compartments to the cell membrane, enhancing or prolonging its signals [17,19], while RET9 is targeted for degradation. Differential ubiquitination, mediated through unique $\mathrm{C}$-terminal interactions, has recently been shown to contribute to these differences in RET isoform processing [16].

\section{RET51 expression is an indicator of more aggressive disease}

Despite the abundance of evidence for distinct roles of the RET isoforms, the implications of RET isoform expression in cancer, and its potential as a marker of disease course, have not been fully explored. In recent studies, we have shown that RET51 modulates multiple critical processes that promote tumor growth and invasive spread in thyroid carcinomas [21]. In both MTC and PTC cell types, RET51 is significantly more effective than RET9 in enhancing cell proliferation, migration and anchorage-independent growth, suggesting that it may be the primary isoform contributing to tumor aggressiveness and disease progression [21]. Consistent with this, RET51 expression is correlated with increased malignancy in human thyroid tumors [21]. RET51 is also more highly expressed relative to RET9 in some MEN2-associated tumors [22] and in breast carcinomas, where it may grant a growth advantage [23]. RET51 is also upregulated in some pancreatic tumors, where it may contribute to invasive potential [24]. Together, these data suggest that RET51 may be the primary oncogenic form of RET in all these tumors. In addition, the relatively higher expression of the RET51 isoform may be an important indicator of RET-mediated tumor invasiveness, overall tumor aggressiveness and poorer prognosis that could identify tumors requiring additional or more extensive treatment interventions. Further, the relative increase in RET51 in these tumors may also make it an attractive target for cancer therapy.

\section{RET as a therapeutic target}

There are currently no approved RET-specific targeted agents, however, several tyrosine kinase inhibitors (TKIs) with activity against multiple kinases have also shown efficacy against RET-expressing cancers. Vandetanib and cabozantinib, multi-kinase TKIs originally developed against VEGFR2, are also effective at inhibiting RET and have been approved for use in patients with advanced MTC or treatment-resistant PTC [4]. In clinical trials, 
TKIs sorafenib, sunitinib and lenvatinib also demonstrate inhibitory activity against both wildtype and mutant RET, and have been identified as options for the treatment of patients with differentiated thyroid carcinomas and MTC [25,26]. More recent innovations in drug design have generated RET-selective TKIs like LOXO-292 (Loxo Oncology), RXDX-105 (Ignyta) and BLU-667 (Blueprint Medicines) that potently inhibit RET over other RTKs, and may provide a valuable new therapeutic tool for use in RET-dependent cancers while avoiding adverse effects associated with broad-spectrum kinase inhibitors [2,26].

Despite the promise of targeted therapy, long-term inhibition of RET signaling may have widespread effects on neuronal maintenance and regeneration or survival, particularly in aged or damaged neurons [27]. Reduced levels of RET activity result in degeneration of dopaminergic neurons and abolishes GDNF-mediated neuroprotective effects in cell and animal models of Parkinson's disease [28], while dysregulation of RET signaling results in nociceptor dysfunction, possibly leading to increases in inflammation-induced pain, as experienced by cancer patients [4]. Importantly, although RET inhibition reduces or stabilizes thyroid tumor size in some patients, tumors quickly develop resistance to these inhibitors and resume growth [29], suggesting the management or maintenance of disease rather than a curative strategy. Despite these drawbacks, the potential of RET-targeted treatments to extend patient life cannot be underestimated and suggests that strategies to further refine the effects of RET inhibition, such as focusing on the primary oncogenic isoform, RET51, could be valuable.

The majority of currently available TKIs recognize the highly conserved sequences of the ATP-binding site of RTKs, allowing them to bind and inhibit multiple kinases. Allosteric inhibitors, which block specific protein interactions or signals, may function as more selective alternatives to existing inhibitors [30]. Targeting of individual RET isoforms or RET isoform-dependent interactions and signaling pathways, using antibodies, peptidomimetics and/or small molecule inhibitors, may offer novel strategies to improve RET selectivity and obviate the adverse events associated with multi-targeting TKIs. Several antibodies that specifically target the RET51 C-terminal tail have been generated and may be effective steric inhibitors to block RET dimerization, autophosphorylation and/or interactions with signaling and adaptor molecules [31]. Peptidomimetics, small peptides that mimic natural substrates, also have the potential for development into potent and selective inhibitors against signaling molecules that bind unique protein domains and motifs [32]. For example, peptides mimicking the sequence and/or structure of the RET C-terminal tails may be useful as 'molecular sponges' to bind RET isoform-specific interactors, such as RET51-binding adaptor GRB2 [4,16,18], thereby inhibiting RET51-specific downstream signals. Alternatively, direct targeting of RET isoforms could be complemented by blocking established signaling pathways activated by RET including ERK/MAPK, PI3K and mTOR [4]. Small molecule inhibitors of these pathways have already been assessed in mechanistic studies and cancer clinical trials for the treatment of thyroid cancer. For example, studies have suggested targeting of the RET-associated PI3K/Akt/mTOR pathway, which is implicated in MTC tumorigenesis and progression and is specifically associated with sustained RET51 activity [17,33]. These novel strategies may provide additional therapeutic options to specifically inhibit RET isoform-dependent functions for the treatment of MTC and PTC or other RET-associated aggressive cancers.

Although work in the field of isoform-specific inhibitors remains nascent, recent studies have identified RET51 as a promising therapeutic target for the treatment of cancers [21]. Ongoing development of therapeutics with greater specificity and the ability to preferentially inhibit this more oncogenic isoform of RET may open new treatment options while avoiding adverse effects associated with current inhibitors.

\section{Financial \& competing interests disclosure}

The authors have no relevant affiliations or financial involvement with any organization or entity with a financial interest in or financial conflict with the subject matter or materials discussed in the manuscript. This includes employment, consultancies, honoraria, stock ownership or options, expert testimony, grants or patents received or pending, or royalties.

No writing assistance was utilized in the production of this manuscript.

\section{Open access}

This work is licensed under the Creative Commons Attribution 4.0 License. To view a copy of this license, visit http://creativecommons.org/licenses/by/4.0/

\section{References}

1. Fagin JA, Wells SA Jr. Biologic and clinical perspectives on thyroid cancer. N. Engl. J. Med. 375(11), 1054-1067 (2016).

2. Wells SA Jr. Advances in the management of MEN2. Endocr. Relat. Cancer 25(2), T1-T13 (2018). 
3. Capdevila J, Galofre JC, Grande E et al. Consensus on the management of advanced radioactive iodine-refractory differentiated thyroid cancer on behalf of the Spanish Society of Endocrinology Thyroid Cancer Working Group (GTSEEN) and Spanish Rare Cancer Working Group (GETHI). Clin. Transl. Oncol. 19(3), 279-287 (2017).

4. Mulligan LM. RET revisited: expanding the oncogenic portfolio. Nat. Rev. Cancer 14(3), 173-186 (2014).

5. Romei C, Ciampi R, Elisei R. A comprehensive overview of the role of the RET proto-oncogene in thyroid carcinoma. Nat. Rev. Endocrinol. 12(4), 192-202 (2016).

6. Wiesner T, He J, Yelensky R et al. Kinase fusions are frequent in Spitz tumours and spitzoid melanomas. Nat. Commun. 5, 3116 (2014).

7. Tahira T, Ishizaka Y, Itoh F, Sugimura T, Nagao M. Characterization of RET proto-oncogene mRNAs encoding two isoforms of the protein product in a human neuroblastoma cell line. Oncogene 5, 97-102 (1990).

8. De Graaff E, Srinivas S, Kilkenny C et al. Differential activities of the RET tyrosine kinase receptor isoforms during mammalian embryogenesis. Genes Dev. 15(18), 2433-2444 (2001).

9. Lee KY, Samy ET, Sham MH, Tam PK, Lui VC. 3' Splicing variants of RET receptor tyrosine kinase are differentially expressed in mouse embryos and in adult mice. Biochim. Biophys. Acta 1627(1), 26-38 (2003).

10. Ivanchuk SM, Myers SM, Mulligan LM. Expression of RET 3' splicing variants during human kidney development. Oncogene 16, 991-996 (1998).

11. Kaplinovsky T, Cunningham AM. Differential expression of RET receptor isoforms in the olfactory system. Neuroscience 175, 49-65 (2011).

12. Tang MJ, Cai Y, Tsai SJ, Wang YK, Dressler GR. Ureteric bud outgrowth in response to RET activation is mediated by phosphatidylinositol 3-kinase. Dev. Biol. 243(1), 128-136 (2002).

13. Enomoto H, Crawford PA, Gorodinsky A, Heuckeroth RO, Johnson EMJ, Milbrandt J. RET signaling is essential for migration, axonal growth and axon guidance of developing sympathetic neurons. Development 128(20), 3963-3974 (2001).

14. Tsui-Pierchala BA, Ahrens RC, Crowder RJ, Milbrandt J, Johnson EM Jr. The long and short isoforms of Ret function as independent signaling complexes. J. Biol. Chem. 277(37), 34618-34625 (2002).

15. Hickey JG, Myers SM, Tian X et al. RET-mediated gene expression pattern is affected by isoform but not oncogenic mutation. Genes Chromosomes Cancer 48(5), 429-440 (2009).

16. Hyndman BD, Crupi MJF, Peng $S$ et al. Differential recruitment of E3 ubiquitin ligase complexes regulates RET isoform internalization. J. Cell Sci. 130(19), 3282-3296 (2017).

17. Richardson DS, Rodrigues DM, Hyndman BD, Crupi MJ, Nicolescu AC, Mulligan LM. Alternative splicing results in RET isoforms with distinct trafficking properties. Mol. Biol. Cell 23(19), 3838-3850 (2012).

18. Alberti L, Borrello MG, Ghizzoni S, Torriti F, Rizzetti MG, Pierotti MA. Grb2 binding to the different isoforms of Ret tyrosine kinase. Oncogene 17(9), 1079-1087 (1998).

19. Crupi MJ, Yoganathan P, Bone LN et al. Distinct temporal regulation of RET isoform internalization: roles of clathrin and AP2. Traffic 16(11), 1155-1173 (2015).

20. Richardson DS, Lai AZ, Mulligan LM. RET ligand-induced internalization and its consequences for downstream signaling. Oncogene 25(22), 3206-3211 (2006).

21. Lian EY, Maritan SM, Cockburn JG et al. Differential roles of RET isoforms in medullary and papillary thyroid carcinomas. Endocr. Relat. Cancer 24(1), 53-69 (2017).

22. Le Hir H, Charlet-Berguerand N, Gimenez-Roqueplo A et al. Relative expression of the RET9 and RET51 isoforms in human pheochromocytomas. Oncology 58(4), 311-318. (2000).

23. Boulay A, Breuleux M, Stephan C et al. The Ret receptor tyrosine kinase pathway functionally interacts with the ERalpha pathway in breast cancer. Cancer Res. 68(10), 3743-3751 (2008).

24. Veit C, Genze F, Menke A et al. Activation of phosphatidylinositol 3-kinase and extracellular signal-regulated kinase is required for glial cell line-derived neurotrophic factor-induced migration and invasion of pancreatic carcinoma cells. Cancer Res. 64(15), 5291-5300 (2004).

25. Mischler K, Kneifel S, Cathomas R. Multitargeted kinase inhibition in metastatic differentiated radioiodine-refractory thyroid cancer: a look at new therapeutic options for a rare disease. Oncol. Res. Treat. 39(9), 548-552 (2016).

26. Redaelli S, Plaza-Manacho I, Lmologni L. Novel targeted therapeutics for MEN 2. Endocr. Relat. Cancer 25(2), T53-T68, (2018).

27. Ibanez CF, Andressoo JO. Biology of GDNF and its receptors - relevance for disorders of the central nervous system. Neurobiol. Dis. 97(Pt B), 80-89 (2017).

28. Drinkut A, Tillack K, Meka DP, Schulz JB, Kugler S, Kramer ER. Ret is essential to mediate GDNF's neuroprotective and neuroregenerative effect in a Parkinson disease mouse model. Cell Death Dis. 7(9), e2359 (2016).

29. Antonelli A, Fallahi P, Ferrari SM et al. New targeted therapies for thyroid cancer. Curr. Genomics 12(8), 626-631 (2011).

30. Bakail M, Ochsenbein F. Targeting protein-protein interactions, a wide open field for drug design. CR Chim. 19(1-2), 19-27 (2016) 
31. Salvatore G, Nagata S, Billaud M, Santoro M, Vecchio G, Pastan I. Generation and characterization of novel monoclonal antibodies to the Ret receptor tyrosine kinase. Biochem. Biophys. Res. Commun. 294(4), 813-817 (2002).

32. Rooklin D, Modell AE, Li H, Berdan V, Arora PS, Zhang Y. Targeting unoccupied surfaces on protein-protein interfaces. J. Am. Chem. Soc. 139(44), 15560-15563 (2017).

33. De Groot JW, Links TP, Plukker JT, Lips CJ, Hofstra RM. RET as a diagnostic and therapeutic target in sporadic and hereditary endocrine tumors. Endocr. Rev. 27(5), 535-560 (2006). 